\title{
Reliability of a new modified tear breakup time method: introduction of dry tear breakup time
}

\author{
Kyung Tae Kim ${ }^{1}$ • Jae-hyung Kim ${ }^{1}$
}

Received: 9 August 2015 / Accepted: 11 August 2015 /Published online: 27 August 2015

(C) Springer-Verlag Berlin Heidelberg 2015

\section{Dear Editor,}

We thank Dr. Sabour for his interest in our article "Reliability of a new modified tear breakup time method: dry tear breakup time"[1]. In this study, WBUT (wet tear breakup time) and DBUT (dry tear breakup time) were measured by one examiner who did not know the purpose of this study. WBUT and DBUT were repeated three times, and each measurement was terminated at 20 seconds to prevent possible corneal drying and other phenomena that might interfere with subsequent measurements. The mean of the three values was then calculated and analyzed. To assess the repeatability of the WBUT and DBUT, an intra-class correlation coefficient (ICC) was used. As has been mentioned, one examiner measured all values so inter-observer reliability couldn't be analyzed. In addition, further evaluation of reliability is most likely needed.

Dr. Sabour further claims that the area under the receiver operating characteristic (AUROC) curve was interpreted incorrectly. However, it is our opinion that most of this criticism lies on the misunderstanding of the expression 'replace' in the abstract of the article. Our intention in this study was not to replace the WBUT method completely with the DBUT method for diagnosing dry eye disease, because an AUROC curve of WBUT was less than that of DBUT. Instead, the intention in this study was to introduce a new modified diagnostic method. However, we do agree with Dr. Sabour claims that further investigation of other estimates for validity is needed.

Conflicts of interest No conflicts of interest have been declared

\section{Reference}

1. Kim KT, Kim JH, Kong YT, Chae JB, Hyung S (2015) Reliability of a new modified tear breakup time method: dry tear breakup time. Graefes Arch Clin Exp Ophthalmol 253(8):1355-1361
Jae-hyung Kim

phaco@mailo.cbnuh.or.kr

1 Department of Ophthalmology, Chungbuk National University

College of Medicine, 776 1st Sunhwan-Ro, Gaesin-dong,

Heungduk-ku, Cheongju 361-711, South Korea 